\title{
Desarrollo de Técnicas de Muongrafía para Estudios Densitométricos de Objetos de Importancia Estratégica
}

\section{Development of Muography Techniques for Densitometric Studies in Objects of Strategic Importance}

Presentación: 6-7/10/2020

\section{Doctorando:}

\section{Rolando Calderón Ardila}

Instituto de Tecnologías en Detección y Astropartículas (ITeDA), Instituto Sabato de Ciencia y Tecnología, UNSAM, Argentina rolando.calderon@iteda.cnea.gov.ar

\section{Director:}

\section{Hernán Asorey}

Co-director:

\section{Alejandro Almela}

\section{Resumen}

La muongrafía es una técnica de prospección no destructiva que permite la determinación de la estructura interna de grandes estructuras tanto naturales como artificiales, por ejemplo volcanes. Esto es posible al construir una imagen con base en la absorción diferencial del flujo direccional de muones atmosféricos producto de la interacción de rayos cósmicos con la atmósfera. Este trabajo doctoral es desarrollado con el propósito principal de aportar en el avance de la muongrafía como técnica de prospección en Geofísica. El trabajo se compone de dos lineas de trabajo, una experimental y una teórica. La experimental se basa en el desarrollo de un prototipo de detector de astropartículas con base en centelladores plásticos y fotomultiplicadores, para esto se usa una electrónica de adquisición desarrollada para el proyecto AMIGA en el Observatorio Pierre Auger y materiales centelladores de plástico. La segunda linea de trabajo trata del estudio del detector y las estructuras de investigación a través de simulaciones computacionales y modelos teóricos. Durante los dos primeros años se completaron los créditos de cursos solicitados por el doctorado, Ademas se trabajó en el estudio de la respuesta de la electrónica, los pulsos, técnicas de análisis de datos y el acople de fotomultiplicadores con los centelladores. Gracias a esto se avanzo en el desarrollo del prototipo y se investigo la posibilidad de incrementar la resolución espacial a través de la detección sincrónica usando electrónicas conectadas al mismo centellador. Actualmente iniciamos el trabajo en los modelos computacionales, estamos estudiando e investigando con respecto a los candidatos a objetos de estudio, destacando el volcán Copahue, volcán Peteroa y la posibilidad de realizar mediciones bajo tierra. 


\section{Abstract}

Muongraphy is a non-destructive survey technique that allows the determination of the internal structure of large natural and artificial structures, such as volcanoes. This is possible by constructing an image based on the differential absorption of the directional flux of atmospheric muons resulting from the interaction of cosmic rays with the atmosphere. This doctoral work is developed with the main purpose of contributing to the advance of muography as a prospecting technique in Geophysics. The work consists of two lines of work, one experimental and one theoretical. The experimental one is based on the development of an astroparticle detector prototype based on plastic scintillators and photomultipliers, using an acquisition electronics developed for the AMIGA project at the Pierre Auger Observatory and plastic scintillator materials. The second line of work deals with the study of the detector and research structures through computer simulations and theoretical models. During the first two years the credits of courses requested by the doctorate were completed. In addition, work was done on the study of the response of electronics, pulses, data analysis techniques and the coupling of photomultipliers with scintillators. Thanks to this, progress was made in the development of the prototype and the possibility of increasing the spatial resolution through synchronous detection between electronics connected to the same scintillator was investigated. Currently we are working on the computer models, we are studying and investigating with respect to the candidates for objects of study, highlighting the Copahue volcano, Peteroa volcano and the possibility of underground measurements.

Keywords: Muography, Particle Detector, Geophysics.

\section{Introducción}

Para estudiar estructuras mediante la técnica de muongrafía, es requerido un instrumento que pueda detectar muones de manera qué, para un tiempo de exposición dado pueda construirse una imagen en términos del contraste de densidad del objeto investigado. Esto significa que el flujo de muones se atenuá al atravesar la materia. Conceptualmente seria similar a una radiografiá, en el caso de la muongrafía la fuente son muones atmosféricos.

Actualmente se han desarrollado y se continua investigando sobre detectores para este propósito, como por ejemplo (Tanaka, 2019) en Japón, (Carbone et al, 2014) en Italia, (Lesparre et al, 2012) en la Isla de Guadalupe en el caribe o (Vesga-Ramírez et al, 2017) en Colombia. Estos detectores son usados para estudiar grandes estructuras tanto naturales como civiles.

Una de las aplicaciones con mayor potencial es en el área de ciencias de la tierra, donde se ha aplicado con éxito en volcanes como en Japón, ver (Tanaka, 2019), actualmente se esta estudiando la evolución del Monte Etna, ver (Presti et al. 2020), otro ejemplo actual es el Volcan Estronboli, ver (Tioukov et al. 2019). Ademas actualmente se realiza caracterización y mediciones del flujo de muones bajo tierra, ver (Zhang et al. 2020) y (Pieczonka et al. 2020). Las tecnologías usadas para estos detectores tienen su origen en la investigación sobre detectores de partículas, donde es requerido censar el paso de estas partículas. En este caso las partículas son los muones que son parte de la familia de los leptones, partículas que tienen carga y $\sim 200$ veces la masa del electrón.

Las diferentes tecnologías de detectores tienen en común el uso de materiales sensibles al paso de partículas cargadas. Algunos diseños están conformados por arreglos de centelladores plásticos como es en nuestro caso, este material es capaz de producir luminiscencia cuando una partícula cargada los atraviesa, sin embargo, en la mayoría de los casos la resolución espacial en las imágenes construidas depende del tamaño de los materiales centelladores. 


\section{Desarrollo}

Este trabajo esta basado en el concepto de transferencia tecnológica, aprovechando la experiencia obtenida del proyecto AMIGA (Pierre Auger Collaboration, 2016) en el Observatorio Pierre Auger en Argentina, el cual desarrolló y construyo detectores capaces de contar muones atmosféricos, en este caso, para estudiar la componente muónica de las cascadas de partículas originadas por rayos cósmicos en la atmósfera.

El desarrollo de este trabajo doctoral implica la construcción de un prototipo constituido por al menos dos planos detectores, de manera que cada uno esta constituido por un arreglo de barras centelladoras cruzadas, cada una de estas barras tiene en su interior una fibra que transporta los fotones hasta el fotomultiplicador, en este caso usaremos Photomultiplier Tube (PMT), por su sigla en Inglés y Silicon Photomultiplier (SiPM), por su sigla en Inglés. En los telescopios de muones construidos con barras de centelleo cruzadas, la resolución de cada panel puede estar dada, entre otras cosas, por la superficie de los centelleadores superpuestos ("píxeles"). Aumentar la resolución fue una de las propuestas iniciales y para lograr esto, se decidió medir y comparar la carga promedio estimada de las medición en ambos extremos de cada barra de centelleo. Así seria posible alcanzar resolución subpíxel obteniendo así una mayor resolución espacial que en los diseños convencionales.
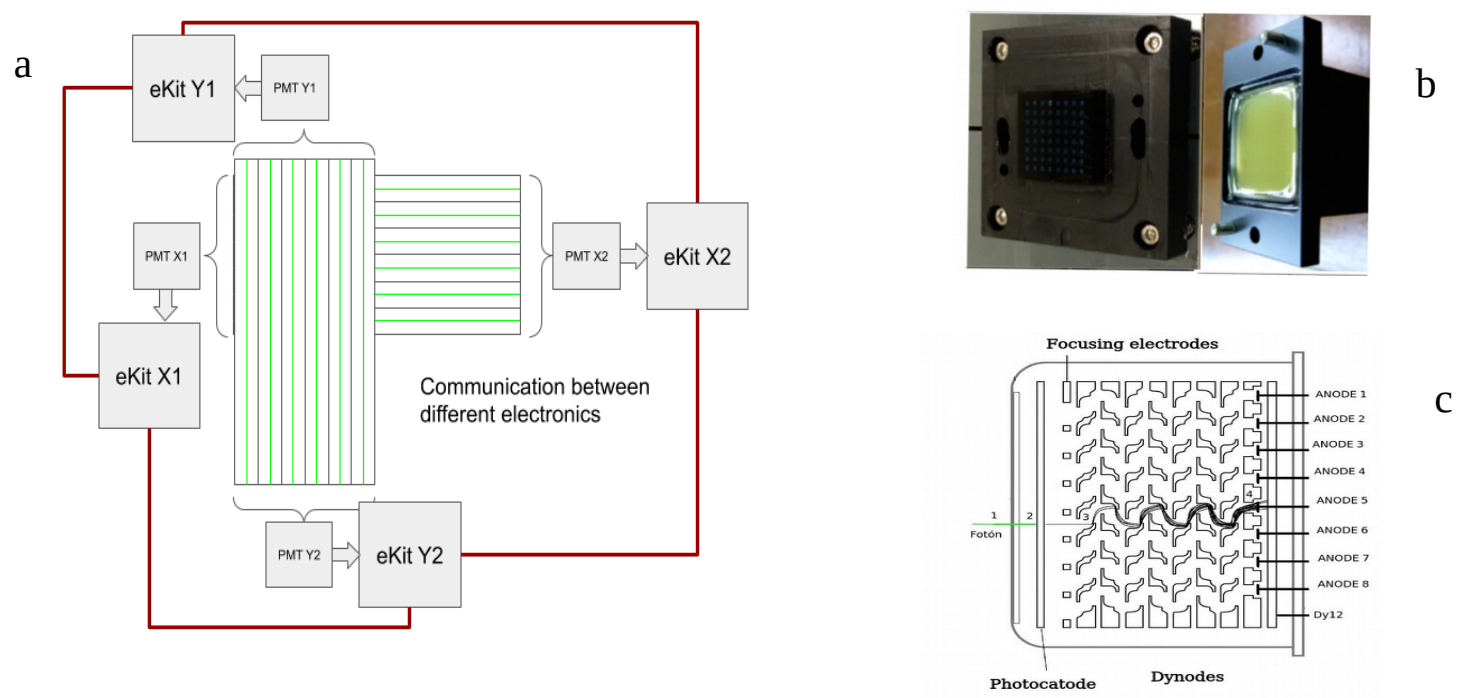

Figura 1: (Izquierda) Esquema de un panel modular, cada par de módulos representa un plano detector. A la derecha podemos observar una imagen de uno de los PMT usados, estos van conectados con acoples mecánicos que los unen a la fibra óptica gracias a un cemento óptico. .

Nuestro primer prototipo utilizara PMTs multi-ánodo de 64 canales H8804-b fabricado por Hamamatsu conectados a través de barras de centellador plástico dopado con PPO [2,5diphenyloxazole] al $1 \%$ y POPOP [1,4-bis(5-phenyloxazole-2-yl)benzene] al 0.03\%, con un recubrimiento de $\mathrm{TiO}_{2}$ Estos centelladores tienen en su interior fibras ópticas de corrimiento de longitud de onda wavelength shifter (WLS), por su sigla en Inglés, estas fibras multi-capa de referencia BCF99-29AMC, tienen un diámetro de 1,2 mm y fueron fabricadas por Saint-Gobain. Cada fibra se conecta a un acople mecánico que une cada extremo de la fibra con el foto-cátodo del PMT que representamos en la Figura 1.b-c. 
Cuando un muon pasa a través del centellador plástico, deposita energía y el centellador la emite en forma de luz, en este caso usamos la unidad de fotones. Estos fotones son absorbidos por la fibra centelladora y re-emitidos en una longitud de onda mas larga, típicamente entre 410 nm y 490nm, la fibra guía los fotones en su interior hasta el acople mecánico donde están en contacto con la superficie del PMT gracias a un cemento óptico que tienen el mismo indice de refracción.

Los fotones que logran llegar al PMT producen una cascada dentro del dispositivo de manera qué produce una corriente generando un pulso. Estos pulsos son identificamos y medidos gracias a la electrónica de adquisición, así medimos y guardamos cada pulso identificándolo como un evento, si cumple ciertos criterios relacionados con el sistema de adquisición. Esta secuencia de detección ha sido probada y es usada por ejemplo, por el proyecto AMIGA (Pierre Auger Collaboration, 2016) en Argentina y el proyecto MuTe (Vásquez-Ramírez et al. 2020) en Colombia.

La primera parte del trabajo que ya fue realizada buscó estudiar como determinar la posición dentro del área de un píxel, para esto se construyo una caja negra o Dark Box en Inglés (ver figura 2), esta caja negra se pensó como un montaje para realizar la calibración de los PMT, cuenta con soportes mecánicos para las electrónicas y una base para sujetar las barras centelladoras, primero concentramos el estudio en la fibra ya que esta en ultima trasporta los fotones a los PMT. Esto se realizo teniendo en cuenta que los centelladores plásticos tienen una longitud de atenuación de alrededor $\sim 4 \mathrm{~cm}$. La fibra se conecto en ambos extremos con PMTs. Luego usamos un láser pulsado Agilent N8042A controlado con una electrónica propia, que emite a $405 \mathrm{~nm}$, emulando la señal esperada en la fibra producida por el centellador. De esta manera pudimos controlar la posición de la fuente, estudiamos y calibramos los PMT, determinamos la carga media depositada a partir del histograma de carga construido con cincuenta mil pulsos por posición. Dado que la fibra posee una longitud de atenuación y una eficiencia de emisión y absorción, la detección se realizó en coincidencia de manera que los dos PMT estuvieran sincronizados con el láser.

Así pudimos comparar las curvas de atenuación y usar esto como parámetro para estimar la ubicación de un evento a lo largo de la fibra. Dado que los píxeles que conformaran cada plano del detector están conformados por barras centelladoras con las fibras embebidas, se logro desarrollar una metodología para mejorar la resolución espacial aplicable a telescopios de muones constituidos de esta manera.

\section{Resultados}

Actualmente estamos trabajando en los modelos computacionales, ademas exploraremos las posibilidades del análisis de datos offline como proponen por ejemplo (Benton et al, 2020), (Vanini et al, 2019) y (Vesga-Ramírez et al, 2020). Hablaremos aquí de las mediciones y avance en el área experimental. En la figura 2 podemos observar el histograma de carga determinado a partir de ciento cuarenta mil muestras obtenidas mediante un osciloscopio (LeCroy WaveSurfer 104MXsB). Para este histograma se obtuvo el mejor ajuste usando un modelo de la respuesta del PMT, que permite comparar la carga depositada en el dínodo de manera que la carga pueda relacionarse como una fracción de la carga medida en un canal individual. Esto abre una nueva posibilidad, ya qué, estudiar esta relación permite proponer otra mejora a partir de la relación de carga, los ánodos permiten ubicar el evento dentro de un píxel, la diferencia de carga depositada entre dínodos permitiría ubicar con mayor precisión la atenuación producida dentro del píxel por la matriz plástica del centellador.

Luego de calibrar el dispositivo se procedió a medir moviendo el láser en diferentes posiciones, generando pulsos y guardando 50.000 en cada posición, una cantidad suficiente para tener una 
buena estadística y se determino el histograma de carga para cada una. Así se obtuvo un ajuste para cada posición con un su respectivo error, donde el valor de carga medio a lo largo de las posiciones representa la atenuación en la fibra WLS,
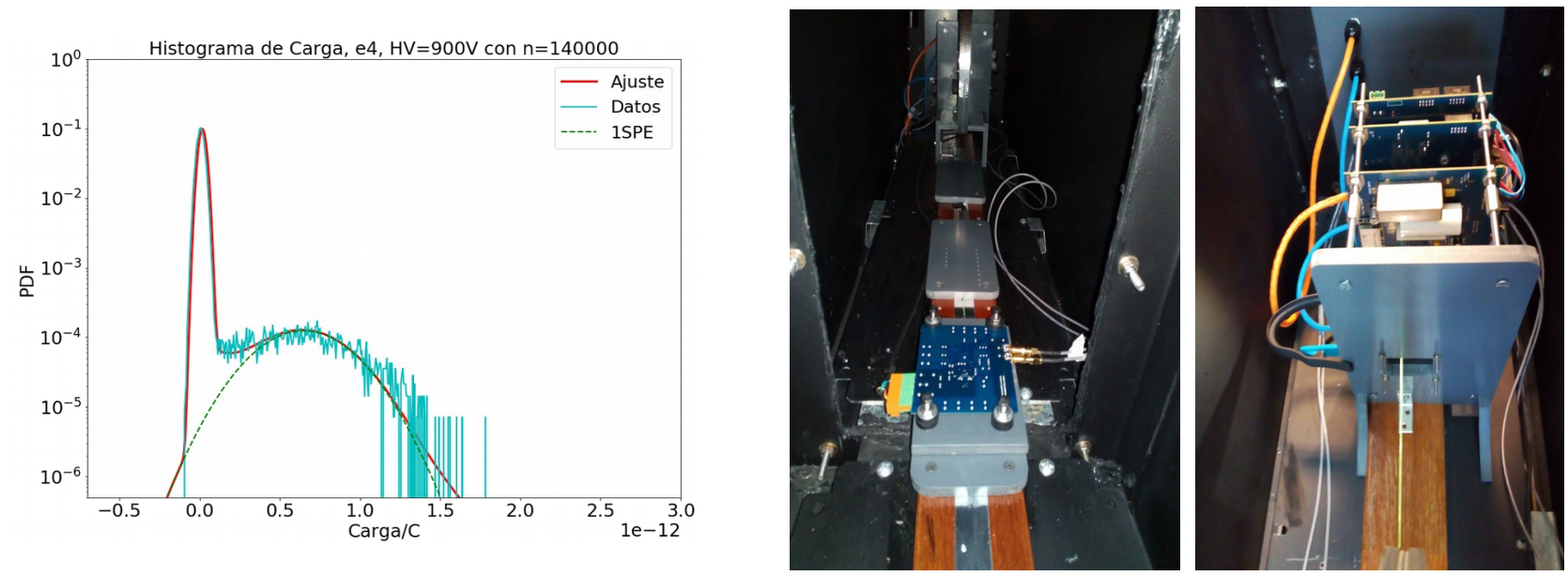

Figura 2: (Izquierda) Histograma de carga para 140000 eventos generados con el láser, para el ajuste se utilizo un modelo de respuesta desarrollado por el Ing Almela en su tesis Doctoral en la UTN. (Derecha) Se puede observar dos imágenes del interior del DarkBox donde se observa las estaciones de acople para el láser, la fibra y los soportes mecánicos con la electrónica de adquisición.

Para cada posición se resto el valor de carga depositada media y se determino el mejor ajuste con sus respectivos intervalos de confianza. Este resultado nos dio información acerca de la incertidumbre con la que podemos determinar la posición de un evento usando este sistema de doble detección, en al figura 3 observamos el ajuste, los intervalos y la proyección del corte para diferentes valores del sigma de error calculado. Presentamos aquí las mediciones para una fibra de $2 \mathrm{~m}$ de longitud, moviendo el láser a lo largo de $120 \mathrm{~cm}$. ver figura 3 . Finalmente las mediciones, calibración y ajustes se presentaron en un congreso ALVO 2020 que se realizo en Chile, se presento el trabajo en el área de instrumentación para prospección Geofísica y adicionalmente se escribió y sometió un paper en la revista Journal of South American Earth Sciences, ver preprint (Calderón-Ardila et al. 2020).

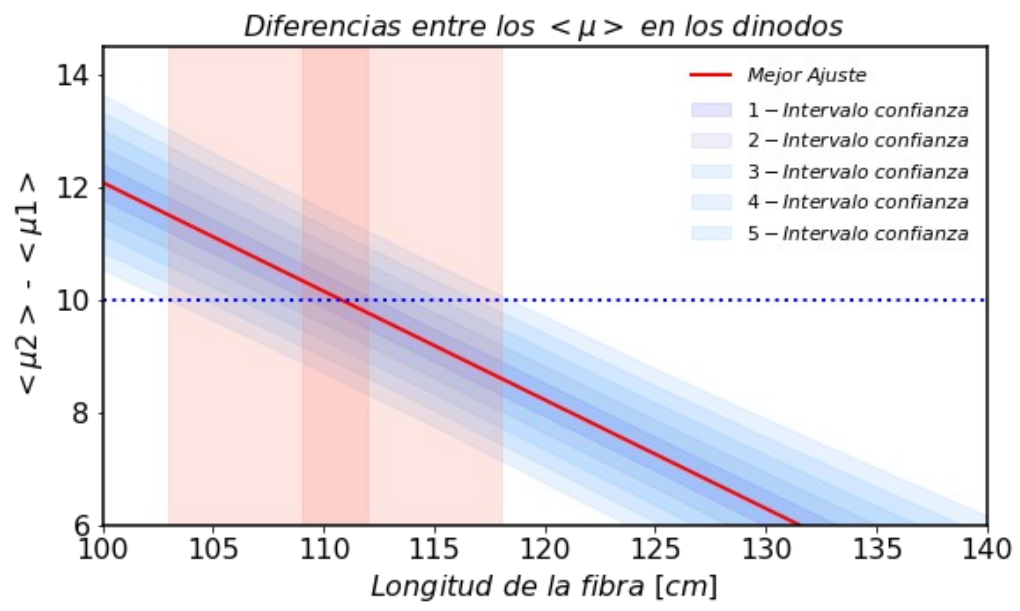

Figura 3 : Ajuste obtenido a partir de los datos medidos a lo largo de la fibra óptica WLS, este grafico tiene un zoom que va desde 100 hasta $140 \mathrm{~cm}$, en azul los intervalos de confianza estimados a partir del error calculado para los datos con respecto al ajuste, en rosado la proyección con respecto al eje $\mathrm{x}$. 


\section{Referencias}

Tanaka, H. K. (2019). Japanese volcanoes visualized with muography. Philosophical Transactions of the Royal Society A, 377(2137), 20180142

Carbone, D., et al. (2014). An experiment of muon radiography at Mt. Etna (Italy). Geophysical Journal International, 196(2):633-643

Lesparre, N., et al. (2012). Density muon radiography of La Soufrière of Guadeloupe Volcano: comparison with geological, electrical resistivity and gravity data. Geophysical Journal International, 190(2):1008-1019

Vesga-Ramírez, A., et al. (2017). Muon Tomography sites for Colombian volcanoes. arXiv preprint arXiv:1705.09884

Pierre Auger Collaboration. (2016). Prototype muon detectors for the AMIGA component of the Pierre Auger Observatory. Journal of Instrumentation, 11(02), P02012

Vásquez-Ramírez, A., et al. (2020). Simulated response of MuTe, a hybrid Muon Telescope. Journal of Instrumentation JINTS, 15(08), P08004

Presti, D., et al. (2020). Muographic monitoring of the volcano-tectonic evolution of Mount Etna. Scientific Reports, 10(1), 1-11

Tioukov, V., et al. (2019). First muography of Stromboli volcano. Scientific reports, 9(1), pg:1-11

Zhang, Z. X., et al. (2020). Muography and Its Potential Applications to Mining and Rock

Engineering. Rock Mechanics and Rock Engineering, 1-15

Pieczonka, S., et al. (2020). On the detectability of density change in steam-assisted gravity drainage reservoirs using muon tomography. The Leading Edge, 39(7), 497-504

Benton, C. J., et al. (2020). Optimizing geophysical muon radiography using information theory. Geophysical Journal International, 220(2), 1078-1094

Vanini, S., et al. (2019). Muography of different structures using muon scattering and absorption algorithms. Philosophical Transactions of the Royal Society A, 377(2137), 20180051

Vesga-Ramírez, A., et al. (2020). Simulated Annealing for Volcano Muography. arXiv preprint, arXiv:2005.08295

Calderón-Ardila, R., et al. (2020). Study of Spatial Resolution of Muon Hodoscopes for Muography Applications in Geophysics. arXiv preprint, arXiv:2006.03165 